\title{
449 - How much frequency do residents fall at nursing homes in Japan ? - THE JADE STUDY
}

Nozomu Oya1, Nobutaka Ayani1, Akiko Kuwahara1, Riki Kitaoka1, Mio Sakuma2, Tsuyoshi Morimoto2, Jin Narumoto1

1 Department of psychiatry, Kyoto Prefectural University of Medicine Japan

2 Department of clinical epidemiology, Hyogo College of Medicine, Japan

Keywords

fall, nursing home, adverse drug event

Introduction

Japan has become an aging society rapidly, and the percentage of the population over 65 was about 28.1\% (around 36 million people) in 2018 in Japan. Then, the number of residents in nursing home has been also increasing.

Many residents in nursing home are receiving pharmacotherapy, and drug-related falls are a major problem at the institution. Fall due to medication sometimes occurs and results in serious consequences.

Then, the aim of this study was to identify the incidence of falls and its association with medication.

Method

The Japan Adverse Drug Event (JADE) study for nursing home is a series of cohort studies. The JADE study for nursing home is a prospective cohort study that was conducted at 4 nursing home for elder people in Japan.

Based on the validated methodology, trained psychologists, medical doctors reviewed all charts to identify ADEs (Adverse Drug Events, injuries due to medication), suspected to being associated with medication. Simultaneously, we collected all falls regardless of drug association.

After collecting these events, 4 medical doctors independently made a secondary review. This study was approved by the institutional review boards of the Kyoto Prefectural University of Medicine.

Result

We enrolled 459 residents, which yielded 3315 resident-months of observation time. The mean (SD) age was 85.8 (7) years and 344 (75\%) were female. We identified 655 falls in 196 residents (39.6\%) during the study period (incidence: 16.9 per 100 resident-months). 568 falls (86.7\%) in 166 residents were related to ADEs. Among them, $10.9 \%$ (62/568) were accompanied by injuries, and $2.6 \%(15 / 568)$ of them resulted in fracture. The most common class of drugs associated with falls was benzodiazepine hypnotics $(23.2 \%, 132 / 568)$ and atypical antipsychotics $(17.4 \%, 99 / 568)$.

\section{Conclusion}

The frequency of falls in nursing homes in Japan was comparable to other countries (L. Z, Rubenstein, 1996). Since significant portion of them were associated with medication, careful monitoring of medication is required. 